\title{
Resection of a dominant-hemisphere intraventricular meningioma facilitated by functional magnetic resonance imaging
}

\author{
Case report
}

\author{
William T. Curry, JR., M.D., G. Rees Cosgrove, M.D., F.R.C.S.(C), \\ Bradley R. BuChbinder, M.D., AND Robert G. OJEMANN, M.D.
}

Neurosurgical Service and Department of Neuroradiology, Massachusetts General Hospital, Harvard Medical School, Boston, Massachusetts

\begin{abstract}
Intraventricular meningiomas of the lateral ventricle occur relatively rarely, but they are often large at the time of detection and present more commonly on the left side. Although the ability to resect these tumors safely has greatly improved over time, standard surgical approaches often traverse cortex close to areas of specific cortical function. Precise cortical mapping of language and sensorimotor cortices can be accomplished noninvasively by using functional magnetic resonance (fMR) imaging. The authors used fMR imaging in planning the cortical incision for resection of a large intraventricular trigone meningioma in the dominant hemisphere of a patient who, postoperatively, suffered no aphasia or hemiparesis. The authors discuss the advantages of mapping cortical function preoperatively with fMR imaging when approaching intraventricular lesions.
\end{abstract}

\section{KEY WORDS • meningioma • lateral ventricle • functional magnetic resonance imaging • cortical mapping}

Since Cushing performed the first radical removal of a meningioma from the lateral ventricle in $1916,{ }^{3}$ the surgical management of these lesions has been a difficult and challenging task for neurosurgeons. A variety of surgical approaches have been used to resect intraventricular meningiomas of the trigone, many of which require transcortical incisions that may be in proximity to eloquent cortex, including sensorimotor and language areas.

Functional MR imaging provides a noninvasive method for identifying human cortical function by using the BOLD technique. This technique is based on the paramagnetic quality of deoxyhemoglobin, which can indirectly reflect neuronal activation due to local changes in regional CBF. Functional MR imaging has been used in a wide variety of neurosurgical applications for the treatment of epilepsy, AVMs, and tumors.

Abbreviations used in this paper: AVM = arteriovenous malformation; $\mathrm{BOLD}=$ blood oxygen level-dependent; $\mathrm{CBF}=$ cerebral blood flow; ECS = electrical cortical stimulation; fMR = functional magnetic resonance; $\mathrm{MR}=$ magnetic resonance; $\mathrm{PET}=$ positron emission tomography.
In our case a meningioma at the trigone of the left lateral ventricle was successfully excised without causing permanent postoperative deficit. Presurgical fMR imaging allowed intraoperative identification of the most appropriate area for cortical incision and allowed us to preserve primary language and sensorimotor cortices. We believe that fMR imaging is a useful adjunct in the preoperative planning for resection of meningiomas of the lateral ventricles and that its role in neurosurgical applications of the future will expand.

\section{CASE REPORT}

Presentation. This 36-year-old right-handed woman presented with difficulty concentrating and mild memory impairment that were chiefly manifest at work. She did not report any significant headache, visual field defect, or language disturbance.

Examination. General physical examination was unremarkable, and a detailed neurological examination demonstrated completely normal results, including visual fields status. Memory and cognition were also normal. 

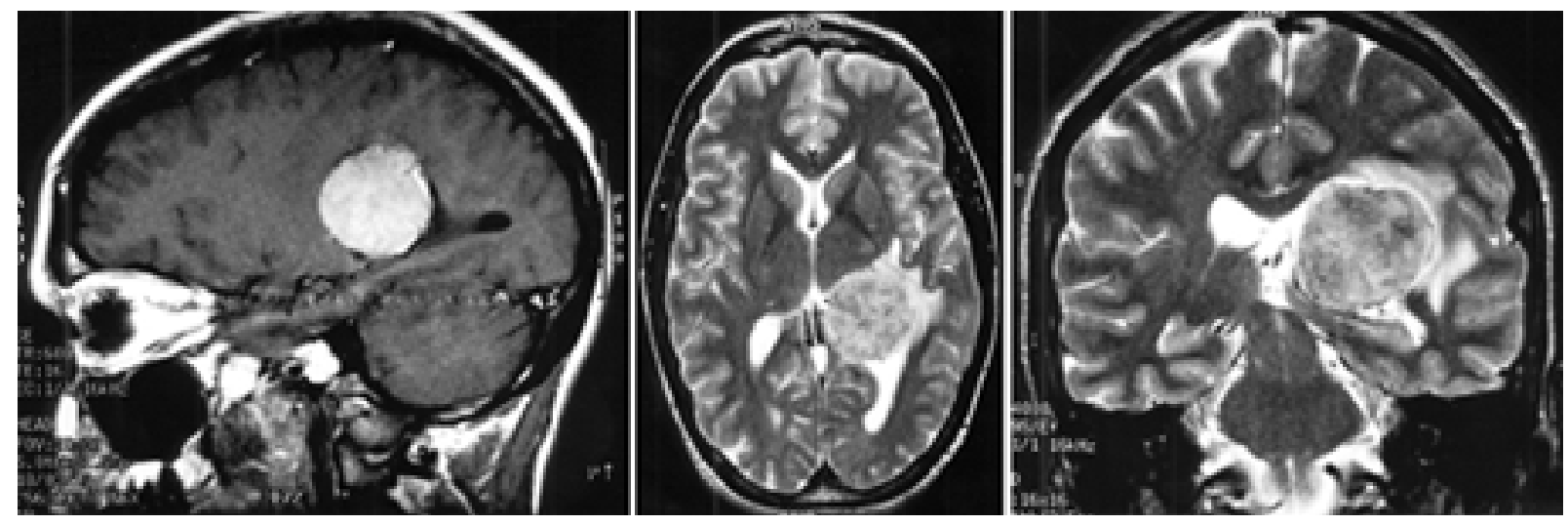

Fig. 1. Left: Sagittal contrast-enhanced $\mathrm{T}_{1}$-weighted MR image demonstrating an enhancing tumor in the left hemisphere. Center and Right: Axial (center) and coronal (right) $\mathrm{T}_{2}$-weighted MR images revealing a large meningioma of the lateral ventricle in the region of the trigone.

Routine MR imaging with and without adminstration of gadolinium revealed a large, well-defined tumor in the left lateral ventricle that enhanced homogeneously (Fig. 1). Angiography revealed a moderately vascular mass with feeding vessels from both the anterior and posterior choroidal arteries. Diagnosis based on radiological findings was consistent with intraventricular meningioma.

Preoperative Cortical Mapping. Functional MR imgaing was performed to identify the sensorimotor and language cortices within the left hemisphere; this was done to plan the optimal surgical approach and select the safest area in which to make a cortical incision (Fig. 2). Structural MR images were first obtained using a volumetric $\mathrm{T}_{1}$-weighted spoiled gradient echo imaging sequence optimized for rendering of cortical anatomy and subcortical disease (TR $25 \mathrm{msec}$, TE $5 \mathrm{msec}$, flip angle $30^{\circ}$, FOV 22, $256 \times 192,1 \mathrm{NEX}$, axial section thickness $1.2 \mathrm{~mm}$ ). Following administration of gadolinium, a second volumetric sequence (TR $28 \mathrm{msec}$, TE $13 \mathrm{msec}$, flip angle $30^{\circ}$ FOV 22, $256 \times 192$, flow compensated, 1 NEX) was obtained to demonstrate cortical veins. Functional data were obtained using a $\mathrm{T}_{2}$-weighted echo planar asymmetrical spin-echo pulse sequence (TR $2000 \mathrm{msec}$, TE 70 msec, offset $25 \mathrm{msec}$, FOV 20, $64 \times 64$ ). The sensorimotor cortex was defined using a hand-activation task that consisted of opening and closing the hand at $2 \mathrm{~Hz}$. The language cortex was defined by a variety of language tasks including verb generation, passive listening, and reading of a complex passage. The three datasets were then coregistered using a surface-matching algorithm and visually rendered for use in the operating room. All imaging was performed on a GE Signa 1.5-tesla imaging system (Milwaukee, WI) by using techniques that have been previously well established at our institution.

The fMR image demonstrated cortical right-hand representation along the central sulcus and language in the operculum of the inferior frontal gyrus and in the posterior superior temporal gyrus on the left (Fig. 2).

Operation. A left, high-parietal approach was chosen because of the size of the lesion and its location in the dominant hemisphere. At surgery, the cortical surface was exposed via a left parietal craniotomy extending to the midline. The cortical gyri and sulci as well as the cortical veins were accurately depicted by the preoperative fMR images. The unique appearance of the cortical veins allowed selection of a cortical zone that respected the presumed language and motor cortices as demonstrated by fMR imaging. The tumor was fully excised through a 2- to $2.5-\mathrm{cm}$ incision at the base of a short parasagittal sulcus, posterior to the sensorimotor cortex, as verified by the fMR image.

Postoperative Course. Postoperatively, she suffered mild confusion and a transient visual field defect but no dysphasia or hemiparesis. At a follow-up visit 10 months after surgery, the patient reported that she was easily fatigued but testing revealed no difficulties with language, improved short-term memory, and full visual field capability. At that time MR imaging revealed no residual tumor.

\section{DISCUSSION}

Although fewer than 5\% of all intracranial meningiomas arise within the ventricular system, the authors of several recent publications have described the clinical and therapeutic challenges of intraventricular meningiomas. ${ }^{8,22}$ The majority (80\%) arise from within the lateral ventricles, whereas $15 \%$ occur in the third ventricle and $5 \%$ in the fourth ventricle. Of lateral ventricular meningiomas, $92 \%$ are found in the region of the trigone and the remainder near the foramen of Monro. ${ }^{2}$ As is the case with all meningiomas, there is a female predominance. Additionally, intraventricular meningiomas exhibit a propensity for growth in the left hemisphere. ${ }^{4}$

Although Cushing and Eisenhardt ${ }^{3}$ attempted to define a clinical syndrome associated with intraventricular meningiomas, no consistent constellation of symptoms truly exists. Almost all patients present with headache, more than half suffer altered visual fields, and approximately $40 \%$ experience dysphagia. ${ }^{8}$ Despite these symptoms, intraventricular meningiomas often become very large before being diagnosed, ${ }^{5}$ rendering resection more formidable.

The relative merits of the various surgical approaches to 


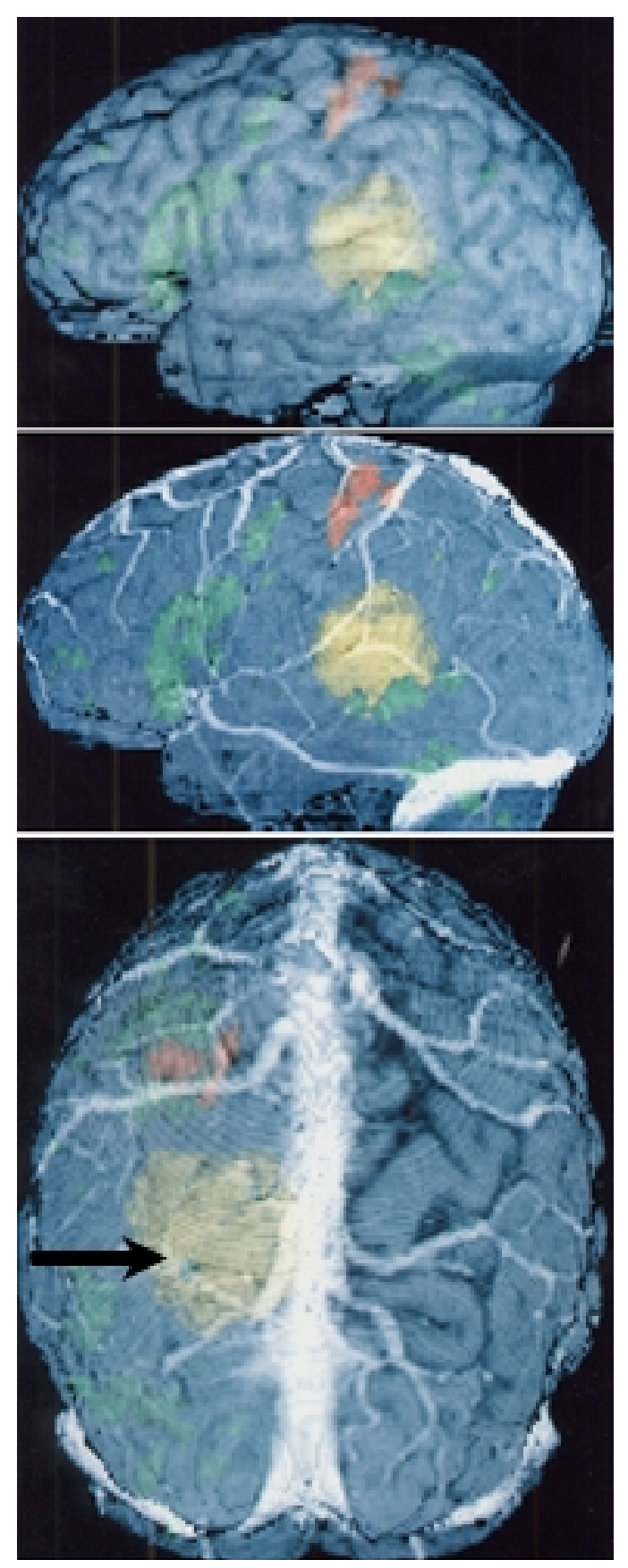

Fig. 2. Upper and Center: Reconstructed three-dimensional renderings in the sagittal plane without (upper) and with (center) cortical veins superimposed, demonstrating the meningioma in yellow, fMR imaging hand activation in red, and language activation in green. Lower: The operative exposure of the left parietooccipital cortex is depicted with the subcortical location of the meningioma seen in relation to the surface. The cortical incision was at the base of the short parasagittal sulcus in the superior parietal lobule, posterior to the area of hand activation (arrow).

meningiomas of the trigone have been debated for several decades. Neurosurgeons have differed in opinion regarding the most efficacious and least destructive approaches to these lesions, particularly with respect to those located in the dominant hemisphere where they occur most frequently. Several factors, including size of the tumor, its blood supply, and the presence of preoperative deficits, must guide the surgeon's choice of where to make the incision.

Cushing was the first to recommend a temporoparietal incision, which provides immediate access to the trigone but virtually assures permanent neurological deficit, most commonly homonymous hemianopia and dysphasia. ${ }^{8}$ The use of a posterior middle temporal gyrus incision generally avoids a complete homonymous visual field defect because the incision is parallel to the fibers of the optic radiations. ${ }^{10}$ In addition, direct access to the vascular pedicle, usually arising from an enlarged and tortuous anterior choroidal artery, is achieved. This approach to tumors in the dominant hemisphere, however, is associated with a high incidence of postoperative language dysfunction. ${ }^{6}$ Likewise, manipulation and retraction may damage posterior superior temporal and supramarginal regions, causing auditory comprehension deficits. ${ }^{23}$

The transcallosal approach is popular among several authors. ${ }^{10,11}$ The incision through the splenium of the corpus callosum seems particularly well suited for smaller tumors that derive their blood supply from the posterior choroidal artery. ${ }^{8}$ Although some purport that partial sectioning of the splenium avoids the rendering of disconnection syndromes, ${ }^{7}$ this risk persists, and alexia without agraphia following transcallosal resection has been reported. ${ }^{14}$

The superior parietooccipital incision used in the patient in our case has proven to be an efficacious and safe approach, particularly for large-sized meningiomas. ${ }^{4}$ As described by Fornari, et al. ${ }^{4}$ this incision extends from the postcentral fissure to the parietooccipital fissure and is 3 to $4 \mathrm{~cm}$ lateral to the falx. In their series, three of 14 patients suffered postoperative weakness and one, who had previously undergone surgery, suffered postoperative language disturbance, although it is not clear that surgery itself was the cause. In addition, four patients sustained new visual field deficits, which the authors attributed to "previous irreversible damage to the optic radiation caused by the tumor itself and/or injury during dissection of the tumor from the ventricular walls." They postulated that this approach should not infringe on the optic radiations because those fibers are lateral and inferior to the ventricle.

An approach of historical significance is the occipital corticotomy/lobectomy, ${ }^{8}$ which carries an absolute risk of homonymous hemianopia and can also lead to dysphasia and dyslexia if extended too far anteriorly. ${ }^{8}$ With improvements in microsurgical techniques over the past two decades, mortality and morbidity rates for resection of intraventricular meningiomas have improved dramatically, with almost all patients experiencing improvement of neurological status in long-term follow up. ${ }^{4,8}$

Functional MR imaging provides a noninvasive means by which to localize cerebral function accurately. The BOLD contrast technique uses the paramagnetic quality of deoxyhemoglobin to record regional $\mathrm{CBF}$ as an indirect measure of neuronal activation. ${ }^{16}$ In response to task performance, fMR imaging delineates the anatomical substrates of cortical function with high spatial resolution, ${ }^{13}$ 
thereby creating an anatomical and functional model of the individual's brain, including rendering of subcortical lesions and their relationships to the cortex.

In addition to an accurate depiction of gyral anatomy and corresponding function, fMR imaging can demonstrate cortical veins, which are effective landmarks that can direct cortical incisions and resection margins. Because the relationship of cortical veins to functional cortex and to subcortical lesions remains intact as the brain undergoes shift after withdrawal of cerebrospinal fluid, following administration of mannitol, or during resection of tumor, these vessels remain important anatomical signposts. An additional feature unique to fMR imaging is its ability to depict the soft tissues and scalp in relation to both underlying functional cortex and subcortical lesions. These surface landmarks can then be used for more logical planning of scalp incisions and removal of bone.

Since 1991, fMR imaging has proven valuable in planning for surgery for tumors ${ }^{21}$ epilepsy, ${ }^{15} \mathrm{AVMs},{ }^{12}$ and cavernous angiomas. ${ }^{1}$ In addition, it has been used in mapping for radiation therapy ${ }^{19}$ and radiosurgery for tumors and AVMs. ${ }^{24}$

Functional MR imaging is both less expensive and potentially more accessible than other noninvasive mapping techniques, including PET, magnetoencephalography, and transcranial magnetic stimulation. Functional MR imaging can be performed using a standard 1.5-tesla magnetic field, and because it does not require injection of radioactive isotopes (as does PET), it is immediately repeatable. To derive structural information from PET, studies must be coregistered with MR images or computerized tomography. With fMR imaging, however, a single imaging modality provides both structural and functional data.

In its current form, fMR imaging has several limitations. Image acquisition occurs over 60 to 90 minutes and requires a specialized staff of physicians and technicians. The modality imaging lacks the ability to localize subcortical white matter tracts, which are of obvious importance in planning a surgical trajectory. Exact knowledge of the limits of the optic radiations, for example, would be of great assistance in approaching meningiomas of the trigone.

For fMR imaging to achieve widespread use, its accuracy in delineating functional cortex must be verified. Although changes in CBF can be detected within seconds, these remain reflections of neuronal activity, whereas electrical techniques are direct measurements. Theoretically, oxygen levels in larger veins that drain diverse and topographically distinct cortical areas change with neuronal activity, which would render BOLD fMR imaging less precise. To avoid this obstacle, fMR imaging must focus on smaller venules and capillaries, which is best achieved using high field strength magnets and spinecho techniques that may not be readily available at many centers.

Electrical cortical stimulation ${ }^{20}$ remains the gold standard for cortical mapping but is invasive, time consuming, and often mandates a more extensive craniotomy than would otherwise be necessary. In addition, because it requires a cooperative, awake patient, ECS is less useful in patients with large mass lesions, when there is increased intracranial pressure, as well as in pediatric patients. Fur- thermore, because ECS can only be performed at the time of surgery, it does not provide functional information for presurgical planning. To date, direct comparison of mapping with fMR imaging with ECS has demonstrated a high degree of congruence between the techniques. ${ }^{9,25}$ This case represents our increasing confidence in the reliability of fMR imaging and its ability to define areas of specific cortical function.

\section{CONCLUSIONS}

Preoperative planning and accurate placement of cortical incisions is essential for the safe resection of meningiomas of the trigone, particularly those in the dominant hemisphere. A number of approaches allows radical resection with relatively little morbidity. Because cortical function may vary in its specific location in different individuals, however, preoperative mapping of functional cortex aids in the choice of surgical approach and placement of cortical incisions. In this case, resection of a large meningioma in the trigone of the left lateral ventricle was performed via a high parietooccipital approach. Accurate placement of the cortical incision was facilitated by preoperative fMR imaging. Both language and sensorimotor cortices were activated by performance of carefully selected tasks, identified preoperatively, and duly preserved. Although the size and location of the lesion remain the most important determinants of approach, specific knowledge of the location of eloquent cortex allows the surgeon greater confidence in where to make the incision. Knowledge of the gross neuroanatomy is essential but not necessarily sufficient because individual variation in cortical function ${ }^{17}$ and distortion or displacement of normal relationships secondary to mass effect can confound even the most accurate and meticulous surgeon. Functional MR imaging at once addresses each of these obstacles as they exist in planning and executing the resection of intraventricular meningiomas. It is, therefore, a valuable asset in the neurosurgical management of these challenging subcortical lesions.

\section{References}

1. Casey AT, Thomas DG, Harkness WF: Stereotactically-guided craniotomy for cavernous angiomas presenting with epilepsy. Acta Neurochir 137:34-37, 1995

2. Criscuolo GR, Symon L: Intraventricular meningioma. A review of 10 cases of the National Hospital, Queens Square (1974-1985) with reference to the literature. Acta Neurochir 83:83-91, 1986

3. Cushing H, Eisenhardt L: Meningiomas: Their Classification, Regional Behavior, Life History, and Surgical End Results. Springfield, IL: Charles C Thomas, 1938

4. Fornari M, Savoiardo M, Morello G, et al: Meningiomas of the lateral ventricles. Neuroradiological and surgical considerations in 18 cases. J Neurosurg 54:64-71, 1981

5. Gassel MM, Davies H: Meningiomas in the lateral ventricles. Brain 84:605-627, 1961

6. Geffen G, Walsh A, Simpson D, et al: Comparison of the effects of transcortical and transcallosal removal of intraventricular tumours. Brain 103:773-778, 1980

7. Geschwind N: Disconnexion syndromes in animals and man. Brain 88:237-294, 1965 


\section{Functional MR imaging-guided resection}

8. Guidetti B, Delfini R: Lateral and fourth ventricular meningiomas, in Al-Mefty O (ed): Meningiomas. New York: Raven Press, 1991, pp 569-582

9. Jack CR Jr, Thompson RM, Butts RK, et al: Sensory motor cortex: correlation of presurgical mapping with functional MR imaging and invasive cortical mapping. Radiology 190:85-92, 1994

10. Jun CL, Nutik SL: Surgical approaches to intraventricular meningiomas of the trigone. Neurosurgery 16:416-420, 1985

11. Kempe LG, Blaylock R: Lateral-trigonal intraventricular tumors. A new operative approach. Acta Neurochir 35:233-242, 1976

12. Latchaw RE, Hu X, Ugurbil K, et al: Functional magnetic imaging as a management tool for cerebral arteriovenous malformations. Neurosurgery 37:619-626, 1995

13. Le Bihan D: Functional MRI of the brain: principles, applications and limitations. J Neuroradiol 23:1-5, 1996

14. Levin HS, Rose JE: Alexia without agraphia in a musician after transcallosal removal of a left intraventricular meningioma. Neurosurgery 4:168-174, 1979

15. Morris GL III, Mueller WM, Yetkin FZ, et al: Functional magnetic resonance imaging in partial epilepsy. Epilepsia 35: 1194-1198, 1994

16. Ogawa S, Lee TM, Kay AR, et al: Brain magnetic resonance imaging with contrast dependent on blood oxygenation. Proc Natl Acad Sci USA 87:9868-9872, 1990

17. Ojemann GA: Individual variability in cortical localization of language. J Neurosurg 50:164-169, 1979

18. Olivecrona H, Lindgren E, Tönnis W, et al: Handbuch de neurochirurgie. Berlin: Springer-Verlag, 1962, Vol 4, pp 175-179
19. Pardo FS, Aronen HJ, Kennedy D, et al: Functional cerebral imaging in the evaluation and radiotherapeutic planning of patients with malignant glioma. Int J Radiat Oncol Biol Phys 30:663-669, 1994

20. Penfield W, Boldrey E: Somatic motor and sensory representation in the cerebral cortex of man as studied by electrical stimulation. Brain 60:389-443, 1937

21. Righini A, deDivitiis O, Prinster A, et al: Functional MRI: primary motor cortex localization in patients with brain tumors. J Comput Assist Tomogr 20:702-708, 1996

22. Schmidek HH (ed): Meningiomas and Their Surgical Management. Philadelphia: WB Saunders, 1991

23. Selnes OA, Knopman DS, Niccum N, et al: Computed tomographic scan correlates of auditory comprehension deficits in aphasia: a prospective recovery study. Ann Neurol 13: $558-566,1983$

24. Witt TC, Kondziolka D, Baumann SB, et al: Preoperative cortical localization with functional MRI for use in stereotactic radiosurgery. Stereotact Funct Neurosurg 66:24-29, 1996

25. Yousry TA, Schmid UD, Jassoy AG, et al: Topography of the cortical motor hand area: prospective study with functional MR imaging and direct motor mapping at surgery. Radiology 195: 23-29, 1995

Manuscript received April 18, 2001.

Accepted in final form May 24, 2001.

Address reprint requests to: G. Rees Cosgrove, M.D., F.R.C.S.(C), Massachusetts General Hospital, 15 Parkman Street, Suite 331, Boston, Massachusetts 02114-2696. email: cosgrove@ helix.mgh.harvard.edu. 